\title{
Maternal intake of vitamins $A, E$ and $K$ in pregnancy and child allergic disease: a longitudinal study from the Danish National Birth Cohort
}

\author{
Ekaterina Maslova $^{1 *}$, Susanne Hansen ${ }^{1}$, Marin Strøm ${ }^{1}$, Thorhallur I. Halldorsson ${ }^{1,2,3}$ and \\ Sjurdur F. Olsen ${ }^{1,4}$ \\ ${ }^{1}$ Centre for Fetal Programming, Department of Epidemiology Research, Statens Serum Institut, Copenhagen, Denmark \\ ${ }^{2}$ Faculty of Food Science and Nutrition, School of Health Sciences, University of Iceland, Reykjavik, Iceland \\ ${ }^{3}$ Unit for Nutrition Research, Landspitali University Hospital, Reykjavik, Iceland \\ ${ }^{4}$ Department of Nutrition, Harvard School of Public Health, Boston, MA, USA
}

(Submitted 24 June 2013 - Final revision received 29 August 2013 - Accepted 18 September 2013 - First published online 11 November 2013)

\begin{abstract}
Fat-soluble vitamins A, E and K have been shown to play roles in immunity and inflammation, but studies on child allergic disease have been few and inconsistent. The aim of the present study was to examine the relationship between maternal intake of vitamins $\mathrm{A}$, $\mathrm{E}$ and $\mathrm{K}$ in mid-pregnancy and child asthma and allergic rhinitis. We used data on 44594 mother-child pairs from the Danish National Birth Cohort. Maternal intake of fat-soluble vitamins was calculated based on the information from a validated FFQ completed in mid-pregnancy. At 18 months, interviews with the mothers were conducted to evaluate doctor-diagnosed child asthma. At age 7 years, we assessed child asthma and allergic rhinitis using questions from the International Study of Asthma and Allergies in Childhood questionnaire and by national registries on hospital contacts and medication use. Current asthma was defined as asthma diagnosis and wheeze in the past 12 months by maternal report. We calculated multivariable risk ratios and $95 \%$ CI by comparing the highest $v$. lowest quintile (Q) of maternal vitamin $\mathrm{A}, \mathrm{E}$ and $\mathrm{K}$ intake in relation to child allergic disease outcomes. Maternal total vitamin $\mathrm{K}$ intake was directly associated with ever admitted asthma (Q5 v. Q1: 1.23, 95\% CI 1.01, 1.50) and current asthma at 7 years (Q5 v. Q1: 1.30, $95 \%$ CI 0.99, 1.70). Weak inverse associations were present for maternal vitamin A and $\mathrm{E}$ intake during pregnancy with child allergic rhinitis. Maternal vitamin $\mathrm{K}$ intake during pregnancy may increase the risk of child asthma, and should be explored further on a mechanistic level. Conversely, maternal vitamin A and $\mathrm{E}$ intake may protect against child allergic rhinitis.
\end{abstract}

Key words: Cohort studies: Pregnancy: Fat-soluble vitamins: Asthma: Allergic rhinitis

Fat-soluble vitamins (D, E, K and A) have in recent years been put on the forefront of asthma and allergy research, primarily by studies on immunomodulatory properties of vitamin D and its association with child wheeze and eczema ${ }^{(1)}$. Maternal vitamin $\mathrm{E}$ intake has likewise been examined in observational studies and found to protect against child wheeze and asthma up to age 5 years $^{(2-4)}$, though results from a recent randomised clinical trial were less convincing ${ }^{(5)}$. The latter null results could be explained by the fact that women were supplemented during the second trimester. It has been suggested that vitamin E influences airway development, and therefore the effect of supplementing vitamin $\mathrm{E}$ might be more pronounced in the first trimester ${ }^{(6,7)}$.

Vitamin A and associated retinoids are important for growth, homeostasis and development ${ }^{(8)}$. Vitamin $\mathrm{A}$ has also been shown to play a prominent role in immune functions.
Animal and in vitro studies have demonstrated how retinoids enhance T-cell proliferation ${ }^{(9)}$ and function in B-lymphocyte growth and development ${ }^{(10)}$. However, few human studies have examined the risk of child asthma in relation to prenatal levels of vitamin A. Vitamin A supplementation in pregnancy has been shown to increase a type 1 immune response ${ }^{(11)}$, suggesting that a skewed in utero milieu may reduce the risk of asthma in the child ${ }^{(12)}$. A randomised clinical trial in Nepal conducted in a vitamin A-deficient population found no relationship with child asthma at 9-13 years of life ${ }^{(13)}$, but found that supplementation improved lung function ${ }^{(14)}$, while in a trial in Indonesia with $\beta$-carotene, children of supplemented mothers had lower levels of interferon- $\gamma^{(15)}$.

Little evidence exists on the role of vitamin $\mathrm{K}$ in asthma development, though new evidence suggests that vitamin $\mathrm{K}$ may have important functions beyond its role in the

Abbreviation: ISAAC, International Study of Asthma and Allergies in Childhood.

*Corresponding author: E. Maslova, email kmv@ssi.dk 
coagulation cascade ${ }^{(16)}$. These include anti-inflammatory properties and free radical-scavenging activity, which may be important in immune system function and development.

Here we aim to replicate current findings on vitamin $\mathrm{E}$ and extend the present analyses to maternal intake of vitamins A and $\mathrm{K}$ from foods and supplements in relation to the risk of child asthma and allergy-related outcomes.

\section{Methods \\ Study population}

The Danish National Birth Cohort enrolled $>100000$ pregnancies between 1996 and 2003 during their first antenatal visit as part of a study to examine pregnancy exposure on maternal and child health during pregnancy and postpartum. Women participated in two interviews during pregnancy (at gestational weeks 12 and 30) and two interviews after birth (at 6 and 18 months). They also filled out a FFQ in gestational week 25 . The participants and their children were followed up through registry linkage using the unique identity number possessed by every Danish citizen and through a selfadministered questionnaire when the children turned 7 years.

Of the 103119 cohort pregnancies, we excluded 4190 multiple births and 303 stillborn singletons, and limited the present data to the first Danish National Birth Cohort pregnancies ( $n$ 84020) in order to avoid dependencies among correlated measures. We had dietary information on 61211 pregnant women and information on supplements from 44772 women. The final dataset was limited to pregnant women with total (diet plus supplement) vitamin A, E and K intake ( $n$ 44594). Sample size varied depending on missing data for the individual outcomes ( -26 to $36 \%$ )

The pregnant women provided written informed consent on behalf of their children. The Regional Scientific Ethics Committee for the municipalities of Copenhagen and Frederiksberg approved all study protocols, and all procedures were in accordance with the Declaration of Helsinki.

\section{Dietary and supplement assessment}

Dietary intake was assessed by using a validated ${ }^{(17,18)} 360$ item FFQ. The questionnaire was mailed to each participant at about week 25 of gestation, and assessed dietary habits and supplement use during the previous 4 weeks $^{(19)}$. In a validation study, estimated intake of retinol was found to correlate well with $7 \mathrm{~d}$ food records ${ }^{(18)}$; vitamin $\mathrm{E}$ and $\mathrm{K}$ intakes were not validated. Daily dietary intakes of vitamins A, E and $\mathrm{K}$ were estimated by multiplying the reported frequencies of consumption with standard portion sizes and recipes and applying information on the content of micronutrients from the National Food Institute's Food Composition Databank (http://www.foodcomp.dk/v7/fcdb_search.asp). Dietary intakes were energy-adjusted using the residual method $^{(20)}$, and women who reported intake that resulted in unrealistic energy intake estimates (arbitrarily set to $<2500$ or $>25000 \mathrm{~kJ} / \mathrm{d}$ ) were excluded (0.5\%). Micronutrient intakes from supplements were calculated from the information that participants provided on the name of the product, the producer, the nutrient content per daily dose and the number of daily doses. We compiled a database of supplement use by combining the information from the Danish Veterinary and Food Administration with the frequency of intake reported by the participants and the daily recommended intake stated on the supplement.

\section{Outcome assessment}

We assessed child asthma status at two different time points using parental reports at 18 months and 7 years and population-based registry data. 'Asthma at 18 months' was defined from the phone interviews as a parental-reported doctor diagnosis. 'Current asthma at 7 years' was defined using standardised core questions from the International Study of Asthma and Allergies in Childhood (ISAAC) ${ }^{(21)}$. To increase the specificity of the 'current asthma at 7 years' outcome, we combined the parental-reported doctor diagnosis and wheezing symptoms in the past 12 months ${ }^{\text {(22) }}$.

In addition to the questionnaire information, we used the data from two registries on hospital contacts and medication use to define asthma diagnoses in the first 7 years of life. These outcomes have been described in detail elsewhere ${ }^{(23)}$. Briefly, we defined 'ever admitted asthma' as children with a first asthma diagnosis in the Danish National Patient Register using the International Classification of Disease 10 codes. Furthermore, we obtained information on the use of antiasthmatic medications from the Register of Medicinal Product Statistics. On the basis of the medication data, we used a validated algorithm to define 'ever prescribed asthma' as children with at least two prescriptions for inhaled $\beta 2$-agonists or steroids or at least one prescription for any other antiasthmatic drug ${ }^{(24)}$

Finally, allergic rhinitis cases were defined as a parental report of a doctor diagnosis of hay fever using an ISAAC question from the 7-year questionnaire.

\section{Covariates}

We collected and evaluated information on maternal sociodemographic and lifestyle covariates from the pregnancy interviews apart from breast-feeding which was based on the interview data from 6 and 18 months postpartum. These covariates included proficiency (by parental education level and occupation), maternal age at birth of child, parity, maternal pre-pregnancy BMI, maternal smoking during pregnancy, maternal exercise during pregnancy, breast-feeding duration, child sex, maternal and paternal history of asthma and allergies, and month of the last menstrual period. Dietary covariates that have previously shown associations with allergic disease were included: fruit and vegetable intake, and intake of long-chain $n$-3 fatty acids, vitamins $\mathrm{D}$ and $\mathrm{C}$, Se and $\mathrm{Zn}$ from diet and supplements (all in quintiles; reviewed in Litonjua $\left.{ }^{(25)}\right)$. All covariates were self-reported except for maternal age and month of the last menstrual period which were extracted from the national registries. The missing indicator method was used to account for missing covariate 
data when the proportion of missing data was $>1 \%$ (socioeconomic status, parity, pre-pregnancy BMI, parental history of asthma and allergies, maternal physical activity and breast-feeding); if $\leq 1 \%$, then the missing observations were deleted $^{(26)}$.

\section{Statistical analysis}

First, we compared the distributions of maternal vitamin A, E and $\mathrm{K}$ intake across the categories of age-standardised covariates. We age-standardised the distribution because of significant differences in maternal vitamin intake across the age categories, with intake among women $\geq 30$ years being higher compared with younger women.

The main exposure was maternal total vitamin intake. In secondary analyses, we further separated out intake from diet and supplements in order to account for potentially different action of food $v$. tablet formulations. Secondary exposures included breaking up vitamin $\mathrm{A}$ intake into $\beta$-carotene and retinol. Maternal vitamin intake was modelled as both linear and by quintiles. To avoid undue influence of outliers in the linear analyses, we restricted the intake to $10000 \mu \mathrm{g} / \mathrm{d}$ for vitamin A ( $n 44$ removed), $1000 \mathrm{mg} / \mathrm{d}$ for vitamin $\mathrm{E}(n 3)$ and $700 \mu \mathrm{g} / \mathrm{d}$ for vitamin $\mathrm{K}(n$ 11) based on either the tolerable upper level or the empirical distribution when the former was missing. Quintiles of exposure were composed when possible and indicator values modelled, comparing each quintile to the lowest (reference) quintile. Furthermore, we examined non-linear relationships for exposures and covariates and OR of child asthma non-parametrically with restricted cubic splines ${ }^{(27)}$. Tests for non-linearity used the likelihood ratio test, comparing the model with only the linear term to that with both the linear and the cubic spline terms. As we have previously found a non-linear relationship between season of the last menstrual period and birth weight with child asthma (E Maslova, unpublished results), we entered these variables as cubic terms into the final model.

Log-binomial models were used to estimate relative risks and $95 \% \mathrm{CI}^{(28,29)}$. In a few instances, the models did not converge and log-Poisson models, which provide consistent, but not fully efficient, estimates of the relative risks and its CI, were used ${ }^{(30)}$. Median values for the exposure categories were entered separately into the models as a continuous variable to evaluate the $P$ value for trend. Interactions by parental asthma and allergy history, breast-feeding and maternal vitamin D intake (vitamin A only) were examined using tertiles of maternal vitamin intake and evaluated using a robust score test ${ }^{(31)}$.

Covariates for the multivariable model were based on biological priors and risk factors for the outcomes identified in the literature. Variables suspected to be on causal pathways, such as gestational weight gain, birth weight, gestational age and child covariates (day-care attendance, antibiotics use in early life and exposure to pets), were initially excluded from the model to avoid overadjustment. Further adjustment for these covariates did not alter the results.

In sensitivity analyses, we dichotomised the exposures according to the $\mathrm{US}^{(32)}$ dietary recommendations. Due to the disagreement of an upper limit for pregnant women ${ }^{(33)}$, we also examined the risk of asthma and allergic rhinitis for vitamin A intake categories of 1000,2000 and $10000 \mu \mathrm{g} / \mathrm{d}$. To determine whether a high or low consumption of all the three vitamins influences the risk of the child outcomes, we composed a fat-soluble vitamin index, grouping women based on whether they were in (1) the lowest quintile of all the three vitamins, (2) the highest quintile of all the three vitamins and (3) any one of the other quintiles (reference). To isolate more clinically relevant asthma diagnoses from the registries rather than more benign wheeze in the first few years of life, we excluded asthma diagnoses that were made during the first 3 years of life ${ }^{(34)}$.

All tests were two-sided, and we used a threshold of $P<0.05$ to denote statistical significance. The analyses were performed using the Statistical Analyses System software (release 9.3; SAS Institute).

\section{Results}

\section{Study population}

Among the pregnant women with information on fat-soluble vitamin intake, about one-half were between the ages 25 and 30 years (45\%), of high or medium proficiency $(53 \%)$ and nulliparous (not having given birth earlier) (56\%). Of the pregnant women, 9 and $32 \%$ reported a history of asthma and allergies, respectively. The median intake of total vitamins A, $\mathrm{E}$ and $\mathrm{K}$ was 1359 (interquartile range 1083-1662) $\mu \mathrm{g} / \mathrm{d}$, 16 (interquartile range $14-18) \mathrm{mg} / \mathrm{d}$ and 122 (interquartile range $80-159) \mu \mathrm{g} / \mathrm{d}$, respectively.

Table 1 shows the age-standardised covariate frequencies across the lowest and highest quintiles of maternal total vitamin $\mathrm{A}, \mathrm{E}$ and $\mathrm{K}$ intake. High-frequency consumers of vitamin A tended to be older, to smoke daily, be multiparous and had marginally lower gestational weight gain. They had a slightly higher vegetable intake, and intake of total folate, $n$ - 3 fatty acids, Se, $\mathrm{Zn}$ and vitamins $\mathrm{D}, \mathrm{K}$ and $\mathrm{C}$, though the standard deviation tended to overlap across the quintiles of maternal vitamin A intake. Similar trends were found for high vitamin $\mathrm{E}$ and $\mathrm{K}$ consumers; however, pregnant women consuming vitamin $\mathrm{E}$ in the highest quintile were less likely to have a BMI $\geq 25 \mathrm{~kg} / \mathrm{m}^{2}$, less likely to report daily smoking and more likely to be physically active. Consumers of vitamin $\mathrm{K}$ in the highest quintile were furthermore less likely to be unskilled and students, less likely to be daily smokers and were more physically active.

Comparing pregnant women with ( $n$ 33415) and without ( $n$ 11 179) outcome data on asthma at 18 months, we found that those with outcome data were more likely to be nulliparous ( $55 v .60 \%)$ and more likely to have children born in the spring and summer (50 v. 44\%). Pregnant women with outcome data at the 7-year time point ( $n$ 28678) $v$. no outcome data ( $n$ 15916) were more likely to be $\leq 25$ years (19 v. 23\%), of high to medium proficiency (55 v. 49\%), have a BMI in the $18.6-24.9 \mathrm{~kg} / \mathrm{m}^{2}$ range $(65 v .60 \%)$ and not smoking (77v. $73 \%)$. 
Table 1. Age-standardised predictors of total vitamin A, E and K intake among 44594 women in the Danish National Birth Cohort ${ }^{\star}$ (Mean values and standard deviations or percentages)

\begin{tabular}{|c|c|c|c|c|c|c|}
\hline \multirow[b]{2}{*}{$\begin{array}{l}\text { Mean }(\mu \mathrm{g} / \mathrm{d}) \\
\text { SD }(\mu \mathrm{g} / \mathrm{d})\end{array}$} & \multicolumn{2}{|c|}{ Total vitamin A quintiles } & \multicolumn{2}{|c|}{ Total vitamin $\mathrm{E}$ quintiles } & \multicolumn{2}{|c|}{ Total vitamin $\mathrm{K}$ quintiles } \\
\hline & $\begin{array}{c}\text { Q1 }(n 8918) \\
838 \\
153\end{array}$ & $\begin{array}{c}\text { Q5 }(n 8919) \\
4515 \\
81877\end{array}$ & 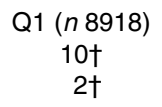 & $\begin{array}{c}\text { Q5 }(n 8919) \\
42 \dagger \\
65 \dagger\end{array}$ & 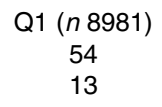 & $\begin{array}{c}\text { Q5 }(n 8981) \\
219 \\
83\end{array}$ \\
\hline \multicolumn{7}{|c|}{ Maternal age (years) $(\%) \ddagger$} \\
\hline$\leq 20$ & 2 & 2 & 2 & 1 & 2 & 1 \\
\hline $20-25$ & 20 & 17 & 21 & 16 & 22 & 15 \\
\hline $25-30$ & 47 & 43 & 44 & 43 & 45 & 44 \\
\hline $30-35$ & 25 & 30 & 26 & 30 & 25 & 30 \\
\hline$>35$ & 6 & 9 & 6 & 10 & 5 & 9 \\
\hline \multicolumn{7}{|c|}{ Socio-economic position (\%) } \\
\hline High & 23 & 22 & 21 & 23 & 20 & 26 \\
\hline Medium & 31 & 29 & 29 & 31 & 29 & 30 \\
\hline Skilled & 25 & 27 & 27 & 25 & 28 & 23 \\
\hline Unskilled & 11 & 13 & 13 & 12 & 13 & 10 \\
\hline Student & 3 & 3 & 3 & 3 & 3 & 4 \\
\hline Unemployed & 1 & 2 & 2 & 1 & 1 & 2 \\
\hline \multicolumn{7}{|c|}{ Pre-pregnancy BMI (\%) $\left(\mathrm{kg} / \mathrm{m}^{2}\right)$} \\
\hline$\leq 18.5$ & 3 & 4 & 3 & 4 & 4 & 4 \\
\hline $18 \cdot 6-24.9$ & 63 & 63 & 61 & 64 & 61 & 66 \\
\hline $25-29 \cdot 9$ & 19 & 18 & 20 & 17 & 19 & 16 \\
\hline$\geq 30$ & 8 & 7 & 8 & 7 & 8 & 7 \\
\hline \multicolumn{7}{|c|}{ Smoking during pregnancy (\%) } \\
\hline Daily smoker & 11 & 13 & 13 & 11 & 14 & 9 \\
\hline \multicolumn{7}{|l|}{ Parity (\%) } \\
\hline Nulliparous & 57 & 49 & 49 & 56 & 50 & 59 \\
\hline \multicolumn{7}{|c|}{ Physical activity (min) (\%) } \\
\hline 0 & 58 & 58 & 61 & 56 & 62 & 52 \\
\hline $1-44$ & 4 & 5 & 4 & 5 & 5 & 5 \\
\hline $45-74$ & 9 & 9 & 8 & 9 & 8 & 9 \\
\hline $75-149$ & 11 & 11 & 10 & 12 & 10 & 13 \\
\hline $150-269$ & 8 & 8 & 7 & 9 & 7 & 10 \\
\hline $270-419$ & 3 & 3 & 2 & 3 & 2 & 3 \\
\hline$\geq 420$ & 1 & 2 & 1 & 2 & 1 & 2 \\
\hline \multicolumn{7}{|l|}{ Maternal asthma (\%) } \\
\hline Yes & 9 & 9 & 9 & 9 & 9 & 9 \\
\hline \multicolumn{7}{|l|}{ Maternal allergy (\%) } \\
\hline Yes & 31 & 30 & 30 & 31 & 30 & 32 \\
\hline \multicolumn{7}{|l|}{ Paternal asthma (\%) } \\
\hline Yes & 8 & 8 & 8 & 8 & 8 & 7 \\
\hline Paternal allergy (\%) & & & & & & \\
\hline Yes & 23 & 23 & 22 & 24 & 23 & 24 \\
\hline Breast-feeding durati & & & & & & \\
\hline No breast-feeding & 2 & 2 & 2 & 2 & 2 & 1 \\
\hline $0-1$ months & 7 & 7 & 7 & 7 & 8 & 5 \\
\hline 2-3 months & 8 & 8 & 8 & 7 & 8 & 7 \\
\hline $4-6$ months & 14 & 13 & 14 & 13 & 15 & 12 \\
\hline $7-9$ months & 20 & 19 & 19 & 19 & 20 & 20 \\
\hline$\geq 10$ months & 22 & 24 & 23 & 25 & 21 & 25 \\
\hline Child sex (\%) & & & & & & \\
\hline Male & 51 & 51 & 51 & 51 & 51 & 51 \\
\hline Gestational weight ga & & & & & & \\
\hline Mean & 479 & 460 & 465 & 470 & 469 & 464 \\
\hline SD & 215 & 217 & 218 & 210 & 215 & 210 \\
\hline Gestational age (d) & & & & & & \\
\hline Mean & 280 & 280 & 281 & 280 & 280 & 280 \\
\hline SD & 12 & 12 & 12 & 12 & 13 & 12 \\
\hline Birth weight $(\mathrm{kg})$ & & & & & & \\
\hline Mean & 3.6 & 3.6 & 3.6 & 3.6 & 3.6 & 3.6 \\
\hline SD & 0.6 & 0.6 & 0.6 & 0.6 & 0.6 & 0.6 \\
\hline Food and nutrient inte & & & & & & \\
\hline Energy (MJ/d) & & & & & & \\
\hline Mean & 9.95 & 9.97 & 9.99 & 9.92 & $10 \cdot 13$ & 9.92 \\
\hline SD & $2 \cdot 63$ & $2 \cdot 76$ & $2 \cdot 68$ & $2 \cdot 74$ & 2.66 & $2 \cdot 72$ \\
\hline Fruit§ (g/d) & & & & & & \\
\hline Mean & 155 & 148 & 129 & 165 & 133 & 174 \\
\hline SD & 108 & 104 & 101 & 112 & 102 & 109 \\
\hline
\end{tabular}


Table 1. Continued

\begin{tabular}{|c|c|c|c|c|c|c|}
\hline \multirow{4}{*}{$\begin{array}{l}\text { Mean }(\mu \mathrm{g} / \mathrm{d}) \\
\text { SD }(\mu \mathrm{g} / \mathrm{d})\end{array}$} & \multicolumn{2}{|c|}{ Total vitamin A quintiles } & \multicolumn{2}{|c|}{ Total vitamin E quintiles } & \multicolumn{2}{|c|}{ Total vitamin $\mathrm{K}$ quintiles } \\
\hline & Q1 ( $n$ 8918) & Q5 ( $n$ 8919) & Q1 ( $n$ 8918) & Q5 ( $n$ 8919) & Q1 ( $n$ 8981) & Q5 ( $n$ 8981) \\
\hline & 838 & 4515 & $10 t$ & $42 \dagger$ & 54 & 219 \\
\hline & 153 & 81877 & $2 \dagger$ & $65 \dagger$ & 13 & 83 \\
\hline \multicolumn{7}{|c|}{ Vegetables\| $(\mathrm{g} / \mathrm{d})$} \\
\hline Mean & 89 & 139 & 95 & 122 & 65 & 180 \\
\hline SD & 59 & 117 & 72 & 99 & 41 & 114 \\
\hline \multicolumn{7}{|c|}{ Alcohol (g/d) } \\
\hline Mean & 1.6 & 1.5 & 1.6 & 1.5 & 1.5 & 1.5 \\
\hline SD & $2 \cdot 2$ & $2 \cdot 1$ & $2 \cdot 4$ & $2 \cdot 1$ & $2 \cdot 4$ & $2 \cdot 1$ \\
\hline \multicolumn{7}{|c|}{ Total $n-3$ fatty acids $(\mathrm{mg} / \mathrm{d})$} \\
\hline Mean & 52 & 75 & 18 & 190 & 59 & 70 \\
\hline SD & 329 & 393 & 196 & 610 & 347 & 382 \\
\hline \multicolumn{7}{|c|}{ Total folate $(\mu \mathrm{g} / \mathrm{d})$} \\
\hline Mean & 568 & 691 & 498 & 745 & 507 & 791 \\
\hline SD & 206 & 283 & 202 & 272 & 202 & 212 \\
\hline \multicolumn{7}{|c|}{ Total vitamin $A(\mu \mathrm{g} / \mathrm{d})$} \\
\hline Mean & - & - & 1070 & 4126 & 1477 & 1574 \\
\hline SD & - & - & 486 & 81931 & 570 & 5613 \\
\hline \multicolumn{7}{|c|}{ Total vitamin $\mathrm{E}(\mathrm{mg} / \mathrm{d})$} \\
\hline Mean & 14 & 27 & - & - & 20 & 24 \\
\hline SD & 24 & 40 & - & - & 39 & 38 \\
\hline \multicolumn{7}{|c|}{ Total vitamin $\mathrm{K}(\mu \mathrm{g} / \mathrm{d})$} \\
\hline Mean & 119 & 127 & 107 & 126 & - & - \\
\hline SD & 50 & 75 & 48 & 74 & - & - \\
\hline \multicolumn{7}{|c|}{ Total vitamin $D(\mu \mathrm{g} / \mathrm{d})$} \\
\hline Mean & 9 & 12 & 8 & 12 & 8 & 14 \\
\hline SD & 6 & 6 & 5 & 6 & 5 & 5 \\
\hline \multicolumn{7}{|c|}{ Total vitamin C $(\mathrm{mg} / \mathrm{d})$} \\
\hline Mean & 201 & 269 & 183 & 288 & 203 & 276 \\
\hline SD & 129 & 165 & 121 & 190 & 131 & 165 \\
\hline \multicolumn{7}{|c|}{ Total Se $(\mu \mathrm{g} / \mathrm{d})$} \\
\hline Mean & 64 & 95 & 55 & 101 & 80 & 86 \\
\hline SD & 23 & 34 & 18 & 32 & 30 & 25 \\
\hline \multicolumn{7}{|c|}{ Total Zn (mg/d) } \\
\hline Mean & 17 & 27 & 16 & 29 & 24 & 23 \\
\hline SD & 5 & 10 & 4 & 9 & 8 & 8 \\
\hline
\end{tabular}

*Values are standardised to the age distribution of the study population.

† Values are expressed in $\mathrm{mg} / \mathrm{d}$.

$¥$ Value is not age adjusted.

§Fruit: citrus fruit + berries + banana + Danish fruit + other fruit.

$\|$ Vegetables: cabbage + onion + green leafy vegetables + root vegetables + tomato + other vegetables.

\section{Parental report of child asthma at 18 months and 7 years}

The prevalence of child asthma at 18 months was 17\% (5632 out of 33415$)$ and $4 \%$ (1122 out of 28678) at 7 years. We did not find any association for maternal vitamin $\mathrm{A}, \mathrm{E}$ and $\mathrm{K}$ intake and child asthma at 18 months, and for maternal vitamin $\mathrm{A}$ and $\mathrm{E}$ intake and current asthma at age 7 years (Tables 2-4 and Tables S1-S6). Maternal dietary vitamin A was related to current asthma at age 7 years $(1 \cdot 20,95 \% \mathrm{CI}$ $1.03,1.39$ for a $1000 \mu \mathrm{g} / \mathrm{d}$ increase) (Table $\mathrm{S} 1$, available online). A higher intake of maternal total vitamin $\mathrm{K}$ was related to an increased risk of current asthma at age 7 years (Q5 v. Q1: 1·30, 95\% CI 0.99, 1·70), which was present already in Q2 (89 (SD 10) $\mu \mathrm{g} / \mathrm{d})$ (Table 4).

\section{Registry-based ever asthma diagnosis at 7 years}

Of the children, 7\% (1983 out of 28678) and 30\% (8738 out of 28678) were classified with asthma by the patient and medication registries, respectively. There was no association for maternal vitamin $\mathrm{A}$ and $\mathrm{E}$ intake with the registry-based asthma outcomes (Tables 2 and 3 and Tables S1, S2, S4 and S5), though maternal vitamin A intake from diet modestly increased the risk of an ever prescribed asthma diagnosis in children (Q5 v. Q1: 1·05, 95\% CI 0·99, 1·11; Table S1, available online). Maternal total vitamin $\mathrm{K}$ intake was directly associated with ever admitted asthma (Q5 $v$. Q1: 1.23, $95 \%$ CI 1.01, 1.50) only; a similar association was present for maternal vitamin $\mathrm{K}$ intake from diet, but not supplements (Table 4; Tables S3 and S6, available online).

To exclude potential transient wheeze in early childhood that may have been captured and classified as asthma by the registries, we excluded the first 3 years of life from these analyses. The number of cases decreased from 1983 to 1014 for ever admitted asthma and from 8738 to 2118 for an ever prescribed asthma diagnosis. The associations were largely unaltered by restricting the analysis to diagnoses above age 3 years. 
Table 2. Associations between maternal total vitamin A intake in mid-pregnancy and child asthma at 18 months and 7 years in the Danish National Birth Cohort (Relative risks (RR) and $95 \%$ confidence intervals)

\begin{tabular}{|c|c|c|c|c|c|c|c|c|c|c|c|c|c|c|c|c|c|c|c|c|}
\hline \multirow[b]{3}{*}{ Quintile of intake } & \multirow[b]{3}{*}{ Cases } & \multirow[b]{3}{*}{$n$} & \multirow{2}{*}{\multicolumn{2}{|c|}{$\begin{array}{c}\begin{array}{c}\text { Asthma } \\
(18 \text { months })\end{array} \\
n^{\star} 33415 / 33105\end{array}$}} & \multirow[b]{3}{*}{$P$} & \multirow[b]{3}{*}{ Cases } & \multirow[b]{3}{*}{$n$} & \multirow{2}{*}{\multicolumn{2}{|c|}{$\begin{array}{c}\begin{array}{c}\text { Current asthma } \\
(7 \text { years })\end{array} \\
n 28678 / 28399\end{array}$}} & \multirow[b]{3}{*}{$P$} & \multirow[b]{3}{*}{ Cases } & \multirow[b]{3}{*}{$n$} & \multirow{2}{*}{\multicolumn{2}{|c|}{$\begin{array}{c}\begin{array}{c}\text { Ever admitted } \\
\text { asthma }\end{array} \\
n 28678 / 28399\end{array}$}} & \multirow[b]{3}{*}{$P$} & \multirow[b]{3}{*}{ Cases } & \multirow[b]{3}{*}{$n$} & \multirow{2}{*}{\multicolumn{2}{|c|}{$\begin{array}{c}\begin{array}{c}\text { Ever prescribed } \\
\text { asthma }\end{array} \\
n 28678 / 28399\end{array}$}} & \multirow[b]{3}{*}{$P$} \\
\hline & & & & & & & & & & & & & & & & & & & & \\
\hline & & & $\mathrm{RR}$ & $95 \% \mathrm{Cl}$ & & & & $\mathrm{RR}$ & $95 \% \mathrm{Cl}$ & & & & $\mathrm{RR}$ & $95 \% \mathrm{Cl}$ & & & & $\mathrm{RR}$ & $95 \% \mathrm{Cl}$ & \\
\hline \multicolumn{21}{|l|}{ Continuoust } \\
\hline Crude & 5626 & 33384 & 1.00 & $0.95,1.04$ & 0.92 & 1121 & 28655 & $1 \cdot 11$ & $1.01,1.22$ & 0.04 & 1980 & 28655 & 1.04 & $0.97,1.13$ & 0.26 & 8733 & 28655 & 1.01 & $0.98,1.04$ & 0.67 \\
\hline \multirow[t]{2}{*}{ Adjusted $\ddagger$} & 5566 & 33077 & 1.01 & $0.96,1.06$ & 0.61 & 1109 & 28377 & 1.08 & $0.97,1.22$ & 0.15 & 1964 & 28377 & 1.07 & $0.98,1.17$ & 0.11 & 8643 & 28377 & 1.01 & $0.97,1.04$ & 0.77 \\
\hline & & & & & $\begin{array}{l}P \text { for } \\
\text { trend§ }\end{array}$ & & & & & $\begin{array}{l}P \text { for } \\
\text { trend§ }\end{array}$ & & & & & $\begin{array}{l}P \text { for } \\
\text { trend§ }\end{array}$ & & & & & $\begin{array}{l}P \text { for } \\
\text { trend§ }\end{array}$ \\
\hline \multicolumn{21}{|l|}{1} \\
\hline Crude & 1105 & 6573 & 1.00 & & 0.52 & 209 & 5553 & 1.00 & & 0.16 & 359 & 5553 & 1.00 & & 0.89 & 1665 & 5553 & 1.00 & & 0.95 \\
\hline \multirow{2}{*}{\multicolumn{21}{|c|}{$2^{2}$}} \\
\hline & & & & & & & & & & & & & & & & & & & & \\
\hline Crude & 1163 & 6661 & 1.04 & $0.96,1 \cdot 12$ & & 240 & 5646 & $1 \cdot 13$ & $0.94,1.35$ & & 438 & 5646 & 1.20 & $1.05,1.37$ & & 1781 & 5646 & 1.05 & $1 \cdot 00,1 \cdot 11$ & \\
\hline \multirow{2}{*}{\multicolumn{21}{|c|}{$0394 \quad 1.1110 .92,1.04$}} \\
\hline & & & & & & & & & & & & & & & & & & & & \\
\hline Crude & 1149 & 6773 & 1.01 & $0.94,1.09$ & & 193 & 5821 & 0.88 & $0.73,1.07$ & & 391 & 5821 & 1.04 & $0.90,1.19$ & & 1736 & 5821 & 0.99 & $0.94,1.05$ & \\
\hline \multirow{2}{*}{\multicolumn{21}{|c|}{$1.080 .93,1.20$}} \\
\hline & & & & & & & & & & & & & & & & & & & & \\
\hline Crude & 1077 & 6655 & 0.96 & $0.89,1.04$ & & 226 & 5878 & 1.02 & $0.85,1.23$ & & 398 & 5878 & 1.05 & $0.91,1.20$ & & 1792 & 5878 & 1.02 & $0.96,1.08$ & \\
\hline Adjusted $\ddagger$ & 1068 & 6589 & 0.98 & $0.89,1.07$ & & 224 & 5820 & 1.02 & $0.83,1.17$ & & 395 & 5820 & $1 \cdot 12$ & $0.95,1.31$ & & 1782 & 5820 & 1.04 & $0.97,1.11$ & \\
\hline \multicolumn{21}{|l|}{5} \\
\hline Crude & 1138 & 6753 & 1.00 & $0.93,1.08$ & & 254 & 5780 & $1 \cdot 17$ & $0.98,1.40$ & & 397 & 5780 & 1.06 & $0.93,1.22$ & & 1764 & 5780 & 1.02 & $0.96,1.08$ & \\
\hline Adjusted $\ddagger$ & 1129 & 6685 & 1.02 & $0.93,1.11$ & & 252 & 5707 & 1.12 & $0.90,1.38$ & & 392 & 5707 & $1 \cdot 10$ & $0.94,1.30$ & & 1736 & 5707 & 1.03 & $0.96,1 \cdot 10$ & \\
\hline
\end{tabular}

*Number of participants in the crude/adjusted analysis.

$\dagger$ Analysis excluded potential outliers as described in the main text. Unit of change was set to $1000 \mu \mathrm{g} / \mathrm{d}$

Adjusted for maternal age, socio-economic status, parity, pre-pregnancy BMI, maternal physical activity, maternal smoking during pregnancy, breast-feeding duration, child sex, maternal history of asthma, maternal history of allergies, paternal history of asthma, paternal history of allergies, season of last menstrual period, and energy, fruit intake, vegetable intake, and intake of fish fatty acids (EPA+DPA + DHA), folic acid, vitamins D and C, Se and

$\S$ Median values for each quintile are entered as a continuous variable into the model. 
Table 3. Associations between maternal total vitamin $E$ intake in mid-pregnancy and child asthma at 18 months and 7 years in the Danish National Birth Cohort (Relative risks (RR) and $95 \%$ confidence intervals)

\begin{tabular}{|c|c|c|c|c|c|c|c|c|c|c|c|c|c|c|c|c|c|c|c|c|}
\hline \multirow[b]{3}{*}{ Quintile of intake } & \multirow[b]{3}{*}{ Cases } & \multirow[b]{3}{*}{$n$} & \multirow{2}{*}{\multicolumn{2}{|c|}{$\begin{array}{c}\begin{array}{c}\text { Asthma } \\
(18 \text { months })\end{array} \\
n^{\star} 33415 / 33105\end{array}$}} & \multirow[b]{3}{*}{$P$} & \multirow[b]{3}{*}{ Cases } & \multirow[b]{3}{*}{$n$} & \multirow{2}{*}{\multicolumn{2}{|c|}{$\begin{array}{c}\begin{array}{c}\text { Current asthma } \\
(7 \text { years })\end{array} \\
n 28678 / 28399\end{array}$}} & \multirow[b]{3}{*}{$P$} & \multirow[b]{3}{*}{ Cases } & \multirow[b]{3}{*}{$n$} & \multirow{2}{*}{\multicolumn{2}{|c|}{$\begin{array}{c}\begin{array}{c}\text { Ever admitted } \\
\text { asthma }\end{array} \\
n 28678 / 28399\end{array}$}} & \multirow[b]{3}{*}{$P$} & \multirow[b]{3}{*}{ Cases } & \multirow[b]{3}{*}{$n$} & \multirow{2}{*}{\multicolumn{2}{|c|}{$\begin{array}{c}\begin{array}{c}\text { Ever prescribed } \\
\text { asthma }\end{array} \\
n 28678 / 28399\end{array}$}} & \multirow[b]{3}{*}{$P$} \\
\hline & & & & & & & & & & & & & & & & & & & & \\
\hline & & & $\mathrm{RR}$ & $95 \% \mathrm{Cl}$ & & & & $\mathrm{RR}$ & $95 \% \mathrm{Cl}$ & & & & $\mathrm{RR}$ & $95 \% \mathrm{Cl}$ & & & & $\mathrm{RR}$ & $95 \% \mathrm{Cl}$ & \\
\hline \multicolumn{21}{|l|}{ Continuous $†$} \\
\hline Crude & 5631 & 33414 & 0.99 & $0.98,1.00$ & 0.10 & 1122 & 28677 & 1.01 & $0.99,1.03$ & 0.36 & 1983 & 28677 & 1.01 & $1.00,1.02$ & 0.06 & 8737 & 28677 & 0.99 & $0.99,1.00$ & 0.17 \\
\hline \multirow[t]{2}{*}{ Adjusted $\ddagger$} & 5572 & 33105 & 1.00 & $0.99,1.01$ & 0.61 & 1110 & 28399 & 1.00 & $0.98,1.02$ & 0.99 & 1967 & 28399 & 1.01 & $1.00,1.02$ & 0.08 & 8648 & 28399 & 0.99 & $0.99,1.00$ & 0.15 \\
\hline & & & & & $\begin{array}{l}P \text { for } \\
\text { trend§ }\end{array}$ & & & & & $\begin{array}{l}P \text { for } \\
\text { trend§ }\end{array}$ & & & & & $\begin{array}{l}P \text { for } \\
\text { trend§ }\end{array}$ & & & & & $\begin{array}{l}P \text { for } \\
\text { trend§ }\end{array}$ \\
\hline \multicolumn{21}{|l|}{1} \\
\hline Crude & 1154 & 6602 & 1.00 & & 0.21 & 208 & 5629 & 1.00 & & 0.17 & 394 & 5629 & 1.00 & & 0.99 & 1685 & 5629 & 1.00 & & 0.69 \\
\hline Adjusted $\ddagger$ & 1144 & 6545 & & & 0.75 & 205 & 5577 & & & 0.80 & 390 & 5577 & & & 0.56 & 1668 & 5577 & & & 0.28 \\
\hline \multicolumn{21}{|l|}{2} \\
\hline Crude & 1159 & 6888 & 0.99 & $0.92,1.07$ & & 256 & 5659 & 1.22 & $1.02,1.46$ & & 427 & 5659 & 1.08 & $0.94,1.23$ & & 1824 & 5659 & 1.08 & $1 \cdot 02,1 \cdot 14$ & \\
\hline Adjusted $\neq$ & 1149 & 6637 & 0.98 & $0.90,1.07$ & & 255 & 5618 & 1.25 & $1.00,1.55$ & & 426 & 5618 & $1 \cdot 11$ & $0.94,1 \cdot 30$ & & 1812 & 5618 & 1.03 & $0.97,1 \cdot 10$ & \\
\hline \multicolumn{21}{|l|}{3} \\
\hline Crude & 1104 & 6697 & 0.94 & $0.87,1.02$ & & 193 & 5748 & 0.91 & $0.75,1 \cdot 10$ & & 369 & 5748 & 0.92 & $0.80,1.05$ & & 1764 & 5748 & 1.03 & $0.97,1.08$ & \\
\hline Adjusted $\ddagger$ & 1089 & 6643 & 0.97 & $0.89,1.06$ & & 192 & 5698 & 0.95 & $0.76,1.20$ & & 366 & 5698 & 0.98 & $0.83,1 \cdot 15$ & & 1748 & 5698 & 1.00 & $0.94,1.07$ & \\
\hline \multicolumn{21}{|c|}{ - } \\
\hline Crude & 1099 & 6741 & 0.93 & $0.87,1.01$ & & 220 & 5987 & 0.99 & $0.83,1.20$ & & 390 & 5987 & 0.93 & $0.81,1.07$ & & 1744 & 5987 & 0.97 & $0.92,1.03$ & \\
\hline Adjusted $\neq$ & 1084 & 6671 & 1.01 & $0.91,1.11$ & & 217 & 5924 & 1.00 & $0.78,1.29$ & & 386 & 5924 & 1.04 & $0.87,1 \cdot 25$ & & 1725 & 5924 & 0.98 & $0.91,1.05$ & \\
\hline \multicolumn{21}{|l|}{5} \\
\hline Crude & 1116 & 6687 & 0.95 & $0.89,1.03$ & & 245 & 5655 & $1 \cdot 17$ & $0.98,1.41$ & & 403 & 5655 & 1.02 & $0.89,1 \cdot 16$ & & 1721 & 5655 & 1.02 & $0.96,1.08$ & \\
\hline Adjusted $\ddagger$ & 1106 & 6609 & 1.01 & $0.91,1.12$ & & 241 & 5582 & 1.06 & $0.82,1.38$ & & 399 & 5582 & 1.07 & $0.88,1.29$ & & 1695 & 5582 & 0.97 & $0.90,1.05$ & \\
\hline
\end{tabular}

Number of participants in the crude/ad

† Analysis excluded potential outliers as described in the main text. Unit of change was set to $10 \mathrm{mg} / \mathrm{d}$.

¥Adjusted for maternal age, socio-economic status, parity, pre-pregnancy BMI, maternal physical activity, maternal smoking during pregnancy, breast-feeding duration, child sex, maternal history of asthma, maternal history of allergies, paternal history of asthma, paternal history of allergies, season of last menstrual period, and energy, fruit intake, vegetable intake, and intake of fish fatty acids (EPA+DPA + DHA), folic acid, vitamins D and C, Se and Zn (all in quintles).

$\S$ Median values for each quintile are entered as a continuous variable into the model. 
Table 4. Associations between maternal total vitamin $\mathrm{K}$ intake in mid-pregnancy and child asthma at 18 months and 7 years in the Danish National Birth Cohort (Relative risks (RR) and $95 \%$ confidence intervals)

\begin{tabular}{|c|c|c|c|c|c|c|c|c|c|c|c|c|c|c|c|c|c|c|c|c|}
\hline \multirow[b]{3}{*}{ Quintile of intake } & \multirow[b]{3}{*}{ Cases } & \multirow[b]{3}{*}{$n$} & \multirow{2}{*}{\multicolumn{2}{|c|}{$\begin{array}{c}\begin{array}{c}\text { Asthma } \\
(18 \text { months })\end{array} \\
n^{\star} 33415 / 33105\end{array}$}} & \multirow[b]{3}{*}{$P$} & \multirow[b]{3}{*}{ Cases } & \multirow[b]{3}{*}{$n$} & \multirow{2}{*}{\multicolumn{2}{|c|}{$\begin{array}{c}\begin{array}{c}\text { Current asthma } \\
\text { (7 years) }\end{array} \\
n 28678 / 28399\end{array}$}} & \multirow[b]{3}{*}{$P$} & \multirow[b]{3}{*}{ Cases } & \multirow[b]{3}{*}{$n$} & \multirow{2}{*}{\multicolumn{2}{|c|}{$\begin{array}{c}\begin{array}{c}\text { Ever admitted } \\
\text { asthma }\end{array} \\
n 28678 / 28399\end{array}$}} & \multirow[b]{3}{*}{$P$} & \multirow[b]{3}{*}{ Cases } & \multirow[b]{3}{*}{$n$} & \multirow{2}{*}{\multicolumn{2}{|c|}{$\begin{array}{c}\begin{array}{c}\text { Ever prescribed } \\
\text { asthma }\end{array} \\
\text { n28678/28399 }\end{array}$}} & \multirow[b]{3}{*}{$P$} \\
\hline & & & & & & & & & & & & & & & & & & & & \\
\hline & & & $\mathrm{RR}$ & $95 \% \mathrm{Cl}$ & & & & $\mathrm{RR}$ & $95 \% \mathrm{Cl}$ & & & & $\mathrm{RR}$ & $95 \% \mathrm{Cl}$ & & & & $\mathrm{RR}$ & $95 \% \mathrm{Cl}$ & \\
\hline \multicolumn{21}{|l|}{ Continuous $†$} \\
\hline Crude & 5632 & 33408 & 0.94 & $0.90,0.98$ & 0.0024 & 1122 & 28671 & 1.03 & $0.94,1 \cdot 13$ & 0.49 & 1982 & 28671 & 1.05 & $0 \cdot 98,1 \cdot 12$ & 0.20 & 8736 & 28671 & 0.97 & $0.94,0.99$ & 0.02 \\
\hline \multirow[t]{2}{*}{ Adjusted $\neq$} & 5572 & 33099 & 0.99 & $0.93,1.05$ & 0.69 & 1110 & 28392 & 1.07 & $0.94,1 \cdot 22$ & 0.33 & 1966 & 28392 & 1.08 & $0.97,1.19$ & 0.15 & 8646 & 28392 & 0.98 & $0.94,1.02$ & 0.39 \\
\hline & & & & & $\begin{array}{l}P \text { for } \\
\text { trend§ }\end{array}$ & & & & & $\begin{array}{l}P \text { for } \\
\text { trend§ }\end{array}$ & & & & & $\begin{array}{l}P \text { for } \\
\text { trend§ }\end{array}$ & & & & & $\begin{array}{l}P \text { for } \\
\text { trend§ }\end{array}$ \\
\hline \multicolumn{21}{|l|}{1} \\
\hline Crude & 1166 & 6691 & 1.00 & & 0.01 & 189 & 5692 & 1.00 & & 0.34 & 372 & 5692 & 1.00 & & 0.10 & 1819 & 5692 & 1.00 & & 0.09 \\
\hline Adjusted $\ddagger$ & 1152 & 6622 & & & 0.82 & 187 & 5613 & & & 0.12 & 369 & 5631 & & & 0.04 & 1798 & 5631 & & & 0.73 \\
\hline \multicolumn{21}{|l|}{2} \\
\hline Crude & 1137 & 6770 & 0.96 & $0.89,1.04$ & & 234 & 5848 & $1 \cdot 21$ & $1 \cdot 00,1 \cdot 45$ & & 380 & 5848 & 0.99 & $0.87,1.14$ & & 1691 & 5848 & 0.90 & $0.86,0.96$ & \\
\hline Adjusted $\ddagger$ & 1126 & 6713 & 1.01 & $0.93,1.09$ & & 230 & 5798 & $1 \cdot 25$ & $1.03,1.52$ & & 373 & 5798 & 1.04 & $0.90,1 \cdot 21$ & & 1676 & 5798 & 0.95 & $0.90,1.01$ & \\
\hline \multicolumn{21}{|c|}{ 等 } \\
\hline Crude & 1144 & 6705 & 0.98 & $0.91,1.05$ & & 249 & 5688 & 1.32 & $1 \cdot 10,1.59$ & & 419 & 5688 & $1 \cdot 13$ & $0.99,1.29$ & & 1788 & 5688 & 0.98 & $0.93,1.04$ & \\
\hline Adjusted $\ddagger$ & 1133 & 6641 & 1.01 & $0.93,1.11$ & & 245 & 5627 & 1.39 & $1 \cdot 12,1.72$ & & 415 & 5627 & $1 \cdot 18$ & $1.01,1.39$ & & 1765 & 5627 & 0.98 & $0.92,1.05$ & \\
\hline \multicolumn{21}{|c|}{ 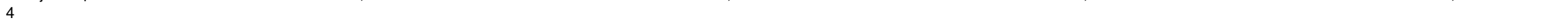 } \\
\hline Crude & 1177 & 6732 & 1.00 & $0.93,1.08$ & & 237 & 5740 & $1 \cdot 24$ & $1.03,1.50$ & & 402 & 5740 & 1.07 & $0.94,1.23$ & & 1754 & 5740 & 0.96 & $0.91,1.01$ & \\
\hline \multirow{2}{*}{\multicolumn{21}{|c|}{ 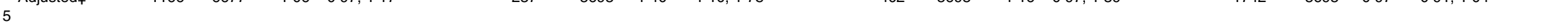 }} \\
\hline & & & & & & & & & & & & & & & & & & & & \\
\hline Crude & 1008 & 6517 & 0.89 & $0.82,0.96$ & & 213 & 5710 & $1 \cdot 12$ & $0.93,1.36$ & & 410 & 5710 & $1 \cdot 10$ & $0.96,1.26$ & & 1686 & 5710 & 0.92 & $0.87,0.98$ & \\
\hline Adjusted $\neq$ & 995 & 6452 & 1.00 & $0.90,1 \cdot 12$ & & 211 & 5648 & $1 \cdot 30$ & $0.99,1 \cdot 70$ & & 408 & 5648 & 1.23 & $1.01,1.50$ & & 1667 & 5648 & 0.97 & $0.90,1.05$ & \\
\hline
\end{tabular}

*Number of participants in the crude/adjusted analysis.

$\dagger$ Analysis excluded potential outliers as described in the main text. Unit of change was set to $100 \mu \mathrm{g} / \mathrm{d}$.

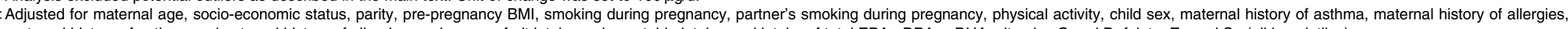

paternal history of asthma and paternal history of allergies, and energy, fruit intake and vegetable intake, and intake of total EPA+DPA + DHA, vitamins C and D, folate, Zn and Se (all in quintiles). 


\section{Parental report of ever child allergic rhinitis}

A total of $5 \%$ (1420 out of 28536) of children were reported by their mother to have a hay fever diagnosis at the 7-year follow-up. Maternal intake of vitamins A, E and $\mathrm{K}$ were not related to ever child allergic rhinitis (Tables S7-S9) however, there was a suggestion towards an inverse association for maternal total vitamin A intake (Q5 $v$. Q1: 0.86, 95\% CI $0 \cdot 70,1 \cdot 04, P=0 \cdot 12)$ and maternal total vitamin $\mathrm{E}$ intake (Q5 v. Q1: 0.84, 95\% CI 0.66, 1.06, $P=0 \cdot 14$ ) (Tables S7 and S8, available online).

\section{Sensitivity analyses}

When examining the relationship between maternal fatsoluble vitamin intake and child asthma and allergic rhinitis using the fat-soluble vitamin index, we found an inverse association for allergic rhinitis only. Consuming in the highest category of the fat-soluble vitamin index ( $n$ 948/13516) decreased the risk of ever child allergic rhinitis at 7 years (all high $v$. middle group: $0 \cdot 60,95 \%$ CI $0.37,0.95$ ).

For maternal vitamin $\mathrm{K}$ intake, meeting the recommended daily intake of $90 \mu \mathrm{g} / \mathrm{d}$ ( $n$ 30191/44594) was associated with an increased risk of current asthma at 7 years (1.31, $95 \%$ CI $1 \cdot 11,1 \cdot 51)$ and ever admitted asthma (1.15, $95 \%$ CI 1.01, 1.30), but there were no associations with the other outcomes; we found no associations for maternal intake of vitamins $\mathrm{A}$ and $\mathrm{E}$.

Since retinol and $\beta$-carotene may affect asthma by different aetiologies, we also examined these exposures separately. Maternal total retinol intake from supplements and diet was inversely associated with child allergic rhinitis only (Q5 (4188 (SD 81 879) $\mu \mathrm{g} / \mathrm{d}) v$. Q1 (632 (SD 146) $\mu \mathrm{g} / \mathrm{d}): 0.79$, 95\% CI $0.65,0.96)$. Maternal dietary $\beta$-carotene intake was directly associated with current asthma at age 7 years (Q5 (6817 (SD 5319) $\mu \mathrm{g} / \mathrm{d}) v$. Q1 (889 (SD 226) $\mu \mathrm{g} / \mathrm{d}): 1 \cdot 38$, $95 \%$ CI 1.06, 1.80).

Examining intake at the high end of the distribution of maternal vitamin A intake (1000, 2000 and $10000 \mu \mathrm{g} / \mathrm{d})$ showed similar inverse associations for retinol, whereas extremely high intakes of $\beta$-carotene seemed to be inversely associated with child asthma.

We analysed the relationship of maternal vitamin A, E and $\mathrm{K}$ intake with any reported wheeze and $>3$ episodes $v$. $\leq 3$ episodes/no wheeze in the first 18 months of the child's life. Maternal vitamin A intake from supplements, but not diet, showed a direct relationship with any wheeze symptoms (Q5 v. Q1: 1·17, 95\% CI 1.05, 1.30). A direct association was also observed between maternal dietary vitamin $\mathrm{E}$ intake and wheeze at 18 months (Q5 v. Q1: 1.06, 95\% CI 0.99, 1.13). For maternal vitamin $K$ intake, no association was observed for wheeze at 18 months, but maternal total vitamin $\mathrm{K}$ intake was directly associated with recurrent wheeze found by comparing Q3 with Q1 (1·19, 95\% CI 1.04, 1·36), but not for other quintiles.

When testing interactions for maternal vitamin A intake and maternal and paternal asthma and allergies, breastfeeding and a vitamin $\mathrm{A} \times$ vitamin $\mathrm{D}$ interaction, we found an increased risk of child asthma at 18 months related to maternal intake of total vitamin A among the children of mothers who reported a history of asthma (Q5 $v$. Q1: 1.21, 95\% CI 0.96, 1.54) and allergies (Q5 v. Q1: 1.18, 95\% CI $1.02,1.37)$, and a decreased risk of ever prescribed asthma related to maternal vitamin $\mathrm{E}$ intake from supplements when mothers breast-fed $\geq 7$ months (Q5 $v$. Q1: 0.87, 95\% CI 0.77, 0.99). We did not find any association in children of mothers who did not report a history of asthma and allergies and those who breast-fed $<7$ months. No interactions were found for maternal vitamin $\mathrm{K}$ intake.

\section{Discussion}

We examined the relationship between maternal intake of vitamins $\mathrm{A}, \mathrm{E}$ and $\mathrm{K}$ during pregnancy and child asthma and allergic rhinitis during the first 7 years of life in 44594 mother-child pairs. We did not find any consistent patterns for maternal vitamin $\mathrm{A}$ and $\mathrm{E}$ intake in relation to child asthma and allergic rhinitis. Maternal vitamin $\mathrm{K}$ intake appeared to increase the risk of ever admitted asthma and current asthma at age 7 years, while maternal vitamin A and $\mathrm{E}$ intake modestly decreased the risk of child allergic rhinitis during the first 7 years of life. Differential associations with child allergic outcomes may be present for maternal retinol $v$. $\beta$-carotene intake. Consuming in the highest quintiles of all the three vitamins decreased the risk of child allergic rhinitis.

The present findings are in conflict with some of the previous studies of fat-soluble vitamins, but support a recent vitamin E supplementation trial ${ }^{(5)}$, which failed to show an effect of high-dose prenatal supplementation with vitamin C and $\mathrm{E}$ on respiratory outcomes at age 2 years. Other studies have found that maternal vitamin $\mathrm{E}$ intake during pregnancy reduced the risk of wheeze in children at the age of 2 years $^{(3,4,35)}$, and noted that these results persisted for wheeze and asthma up to the age of 5 years ${ }^{(2)}$. Other studies have reported both an increase in wheeze and no association $^{(36,37)}$. Comparison between studies should be done with care due to differences in study design and populations, and speculations regarding the potential threshold level of vitamin $\mathrm{E}$ intake for optimal immunity are difficult since some studies have not reported the levels of vitamin $\mathrm{E}$ intake in exposure groups ${ }^{(2,35)}$. We are aware of only one study that related maternal vitamin $\mathrm{E}$ intake during pregnancy to child allergic rhinitis and found no association ${ }^{(2)}$. Studies on food sources of vitamin $\mathrm{E}$ in pregnancy and allergic rhinoconjunctivitis/rhinitis in children have found both inverse ${ }^{(38)}$ and direct $^{(39)}$ associations. Maternal vitamin E supplementation has not demonstrated any benefit in improving the symptoms of seasonal or perennial allergic rhinitis in the offspring ${ }^{(40,41)}$.

Studies in infants have indicated that vitamin E may activate a type 1 immune response rather than an asthma-inducing type 2 response ${ }^{(42,43)}$. Since early-life respiratory symptoms may include both transient wheeze due to respiratory infection and true asthma, it could explain previous findings of protective associations with wheeze at $<2$ years of age and our own null finding for child asthma at 7 years of age. 
However, the present study suggests that high maternal vitamin $\mathrm{E}$ intake during pregnancy may modestly protect against child allergic rhinitis.

Few studies have examined maternal vitamin A supplementation in relation to allergic diseases. Consistent with our null finding, a trial in Nepal that supplemented women with $7000 \mu \mathrm{g}$ retinol equivalents (RE) of provitamin A, $42 \mathrm{mg}$ $\beta$-carotene or placebo during pregnancy has found no association with child asthma incidence, but showed improved lung function with supplementation ${ }^{(13,14)}$. A randomised trial among Indonesian women on $\beta$-carotene supplementation has noted lower interferon- $\gamma$ production among their infants $^{(15)}$. In the present study, we found that a high intake of $\beta$-carotene decreased the risk of asthma, and these findings were strongest for maternal doses $\geq 10000 \mu \mathrm{g} / \mathrm{d}$. This suggests that both the quantity and quality of vitamin A may be important to consider or that the risk may vary by study population. Studies in infants have found that vitamin A-deficient infants have significantly lower interferon $-\gamma$ production compared with infants with adequate vitamin A status ${ }^{(44)}$. Animal studies have found opposite results, in which vitamin A deficiency has been reported to decrease the development of experimental asthma $^{(45,46)}$, suggesting that deficiency may skew towards a type 1 response and away from a more asthma-inducing type 2 response. An up-regulation of a type 1 response with vitamin A deficiency was also observed among children aged $<13$ years examined cross-sectionally ${ }^{(47)}$. On the contrary, a study in pregnant women supplemented with retinol ( $v$. placebo) has found increased IL-10 and interferon- $\gamma$ production, skewing away from a type 2 response ${ }^{(11)}$. However, it is unclear whether these changes are sufficient to influence the development of the fetal immune system. The present results showed no increase in child asthma risk with a higher maternal intake of vitamin A, and only a weak increase for an ever prescribed asthma diagnosis. The differences in findings may include a difference in underlying vitamin A intake and biological levels where a change from a deficient or near-deficient state may have a positive impact on fetal respiratory and immune system development, while excess vitamin A from a sufficient state may have minor negative consequences. However, adverse effects cannot be excluded for other endpoints ${ }^{(33)}$. Additionally, a family history of asthma and allergies may modify the risk of early asthma in the offspring.

We found that the risk of child allergic rhinitis was reduced with a higher maternal intake of vitamin A, specifically retinol. Studies in children have found a reduced risk of allergic rhinitis and associated symptoms with vitamin A sources such as vegetables ${ }^{(38)}$ and milk ${ }^{(39)}$, but not vitamin A itself $^{(38,48)}$. The inverse results found for maternal vitamin A and (indicative for) $\mathrm{E}$ intake in the present study may point to the importance of these nutrients in the prevention of sensitisation (e.g. T-helper cell switching, IgE production) rather than direct respiratory effects.

We initially hypothesised that proposed anti-inflammatory properties and free radical-scavenging activities of vitamin $\mathrm{K}_{16}$ may lead to a reduced risk of asthma and allergic rhinitis in children whose mothers ate at the higher end of the distribution. The results of the present study indicated the opposite, that maternal total vitamin $\mathrm{K}$ intake increases the risk of ever admitted asthma and current asthma in children at age 7 years. To our knowledge, this is the first study to examine maternal vitamin $\mathrm{K}$ intake during pregnancy in relation to these outcomes and hence give us no prior evidence on which to draw from. Despite the many beneficial effects of vitamin K on human health, since the 1980s, there have been several case reports of hypersensitivity and anaphylactic reactions to intravenous $^{(49-54)}$ and cutaneous ${ }^{(55-57)}$ vitamin $\mathrm{K}$ injections. While rare and short-enduring, these and the present data may point to a greater and more complex involvement of vitamin $\mathrm{K}$ in sensitisation and related diseases.

There are some limitations to the present study. Dietary assessment using FFQ results in measurement error due to assumptions on portion sizes, quality of food tables and accuracy in self-reporting. Dietary measurement error is usually random and would therefore only underestimate the strength of any true underlying association to the null. In the present analysis, we were not able to adjust for exposure of child vitamin $\mathrm{A}, \mathrm{E}$ and $\mathrm{K}$ intake during breast-feeding and in childhood due to the lack of this information; we can therefore not exclude the possibility that maternal diet served as a marker for diet during breast-feeding. However, both observational and supplementation studies have not been able to link either child intake of vegetables or supplements with asthma $^{(13,58-61)}$, indicating that any confounding by child diet is most probably minor or negligible. Observational studies with a long follow-up are prone to both confounding and selection bias. We cannot exclude the possibility of residual confounding by unmeasured or mismeasured covariates; nonetheless, by adjusting for numerous covariates, we limited the possibility of residual confounding. We also examined the distributions of sociodemographic and lifestyle covariates among participants and non-participants of the present study and found few differences, suggesting that any present selection bias would be limited

The present study has several strengths. We were able to follow nearly 45000 mother-child pairs prospectively and longitudinally. The FFQ used in the present study assessed diet in great detail, allowing us to capture maternal vitamin intake from both diet and supplements, as well as focus on both pre- and provitamin A in order to discern potential aetiological differences. The extensive dietary questionnaire would also more accurately assess energy intake and reduce measurement error when dietary intake is energy-adjusted.

Asthma and allergic disease have a time-dependent manifestation of symptoms ${ }^{(62-64)}$. While wheeze and 'asthma' in the first few years of life may represent transient conditions due to viral infections, respiratory and sensitisation symptoms by age 7 years are more likely to represent clinical disease ${ }^{(63)}$ Following the children from conception to 7 years of age, we examined numerous outcomes stretching from early to later childhood, and thereby investigating possible associations with several phenotypes of asthma that may be governed by different aetiologies. In addition to self-reported measures of asthma that would be an overall measure of asthma and would not specifically distinguish between phenotypes of 
asthma, we were able to include two additional measures based on population-based register data. Admitted asthma represents a more severe phenotype or uncontrolled asthma that required treatment in a hospital setting, while prescribed asthma is more likely to be indicative of mild to moderate asthma requiring some form of medication. For both these registry-based outcomes, the majority of diagnoses were made $<3$ years and may therefore not represent a permanent asthmatic phenotype, warranting, as in the present analysis, the examination of cases appearing only after the age of 3 years. For allergic rhinitis, we only had access to one ISAAC question at 7 years relating to an ever doctor diagnosis of hay fever. This question does not capture current or seasonal symptoms, but has been validated against skin prick tests, and self-reported hay fever has been shown to agree well with the symptoms of allergic rhinitis and atopy ${ }^{(65,66)}$.

In conclusion, in the present large prospective cohort of Danish mothers and children, we found no overall pattern of association between maternal intake of fat-soluble vitamins and child allergic disease; yet, maternal intake of vitamin $\mathrm{K}$ during pregnancy increased the risk of ever admitted asthma. Weak inverse associations were suggested for maternal total vitamin A and $\mathrm{E}$ intake with ever child allergic rhinitis. The results suggest differential associations for sources of intake as well as type of allergic outcome. These findings, especially for the less studied vitamin $\mathrm{K}$, need to be replicated in different cohorts and mechanisms explored in animal studies.

\section{Supplementary material}

To view supplementary material for this article, please visit http://dx.doi.org/10./S0007114513003395

\section{Acknowledgements}

We thank Anne Ahrendt Bjerregaard for initial background research on maternal vitamin $\mathrm{E}$ intake and child asthma.

The present study was supported by the Danish Council for Strategic Research (09-067124); the Danish Council for Independent Research/Medical Sciences, Danish Agency for Science, Technology and Innovation (09-063410); the Lundbeck foundation (R13-A907); and the European Union Integrated Research Project EARNEST (FOOD-CT-2005007036). The European Union project EARNEST (http:// www.metabolic-programming.org) received financial support from the Commission of the European Communities under the FP 6 priority 5: food quality and safety. The Danish National Birth Cohort was financed by the March of Dimes Birth Defects Foundation, the Danish Heart Association, the Danish Medical Research Council, the Sygekassernes Helsefond, the Danish National Research Foundation, the Danish Pharmaceutical Association, the Ministry of Health, the National Board of Health and Statens Serum Institut. The supporting bodies for this project had no role in the design, analysis or writing of this article.

The authors' responsibilities were as follows: E. M. was involved in the study concept and design, prepared the data and performed the analyses; E. M., S. H. and M. S. conducted the main statistical analyses; E. M. and S. H. drafted the manuscript; E. M., S. H., M. S., T. I. H. and S. F. O. contributed to the critical advice and the revision of the manuscript; S. F. O. obtained funding; E. M. and S. F. O. had a role in the acquisition of the data and had responsibility for the entire content of the manuscript. All authors had full access to the study data.

None of the authors had a conflict of interest.

\section{References}

1. Litonjua AA (2012) Fat-soluble vitamins and atopic disease: what is the evidence? Proc Nutr Soc 71, 67-74

2. Devereux G, Turner SW, Craig LC, et al. (2006) Low maternal vitamin $\mathrm{E}$ intake during pregnancy is associated with asthma in 5-year-old children. Am J Respir Crit Care Med 174, 499-507.

3. Litonjua AA, Rifas-Shiman SL, Ly NP, et al. (2006) Maternal antioxidant intake in pregnancy and wheezing illnesses in children at 2 y of age. Am J Clin Nutr 84, 903-911.

4. Miyake Y, Sasaki S, Tanaka K, et al. (2010) Consumption of vegetables, fruit, and antioxidants during pregnancy and wheeze and eczema in infants. Allergy 65, 758-765.

5. Greenough A, Shaheen SO, Shennan A, et al. (2010) Respiratory outcomes in early childhood following antenatal vitamin C and E supplementation. Thorax 65, 998-1003.

6. Gonzalez-Reyes S, Martinez L, Martinez-Calonge W, et al. (2006) Effects of nitrogen and vitamins A, C and E on maturation of cultured human H441 pneumocytes. Biol Neonate 90, 9-16.

7. Islam S, Narra V, Cote GM, et al. (1999) Prenatal vitamin E treatment improves lung growth in fetal rats with congenital diaphragmatic hernia. J Pediatr Surg 34, 172-176, (discussion $6-7)$

8. Allende LM, Corell A, Madrono A, et al. (1997) Retinol (vitamin A) is a cofactor in CD3-induced human T-lymphocyte activation. Immunology 90, 388-396.

9. Friedman A, Halevy O, Schrift M, et al. (1993) Retinoic acid promotes proliferation and induces expression of retinoic acid receptor-alpha gene in murine $\mathrm{T}$ lymphocytes. Cell Immunol 152, 240-248.

10. Blomhoff HK, Smeland EB, Erikstein B, et al. (1992) Vitamin A is a key regulator for cell growth, cytokine production, and differentiation in normal B cells. $J$ Biol Chem 267, 23988-23992.

11. Cox SE, Arthur P, Kirkwood BR, et al. (2006) Vitamin A supplementation increases ratios of proinflammatory to anti-inflammatory cytokine responses in pregnancy and lactation. Clin Exp Immunol 144, 392-400.

12. Holgate ST (2004) The epidemic of asthma and allergy. J Royal Soc Med 97, 103-110.

13. Checkley W, West KP Jr, Wise RA, et al. (2011) Supplementation with vitamin A early in life and subsequent risk of asthma. Eur Respir J 38, 1310-1319.

14. Checkley W, West KP Jr, Wise RA, et al. (2010) Maternal vitamin A supplementation and lung function in offspring. $N$ Engl J Med 362, 1784-1794.

15. Wieringa FT, Dijkhuizen MA, Muhilal, et al. (2010) Maternal micronutrient supplementation with zinc and beta-carotene affects morbidity and immune function of infants during the first 6 months of life. Eur J Clin Nutr 64, 1072-1079.

16. Shearer MJ \& Newman P (2008) Metabolism and cell biology of vitamin K. Thromb Haemost 100, 530-547.

17. Mikkelsen TB, Olsen SF, Rasmussen SE, et al. (2007) Relative validity of fruit and vegetable intake estimated by the food 
frequency questionnaire used in the Danish National Birth Cohort. Scand J Public Health 35, 172-179.

18. Mikkelsen TB, Osler M \& Olsen SF (2006) Validity of protein, retinol, folic acid and $n-3$ fatty acid intakes estimated from the food-frequency questionnaire used in the Danish National Birth Cohort. Public Health Nutr 9, 771-778.

19. Olsen SF, Mikkelsen TB, Knudsen VK, et al. (2007) Data collected on maternal dietary exposures in the Danish National Birth Cohort. Paediatr Perinat Epidemiol 21, 76-86.

20. Willett WC (1998) Nutritional Epidemiology, 2nd ed. New York, NY: Oxford University Press.

21. Asher MI, Keil U, Anderson HR, et al. (1995) International study of asthma and allergies in childhood (ISAAC): rationale and methods. Eur Respir J 8, 483-491.

22. Hederos CA, Hasselgren M, Hedlin G, et al. (2007) Comparison of clinically diagnosed asthma with parental assessment of children's asthma in a questionnaire. Pediatr Allergy Immunol 18, 135-141.

23. Hansen S, Strom M, Maslova E, et al. (2012) A comparison of three methods to measure asthma in epidemiologic studies: results from the Danish National Birth Cohort. PLOS ONE 7 , e36328.

24. Moth G, Vedsted P \& Schiøtz PO (2007) Identification of asthmatic children using prescription data and diagnosis. Eur J Clin Pharmacol 63, 605-611.

25. Litonjua AA (2008) Dietary factors and the development of asthma. Immunol Allergy Clin North Am 28, 603-629, ix.

26. Greenland S \& Finkle WD (1995) A critical look at methods for handling missing covariates in epidemiologic regression analyses. Am J Epidemiol 142, 1255-1264.

27. Durrleman S \& Simon R (1989) Flexible regression models with cubic splines. Stat Med 8, 551-561.

28. Wacholder S (1986) Binomial regression in GLIM: estimating risk ratios and risk differences. Am J Epidemiol 123, $174-184$

29. Skov T, Deddens J, Petersen MR, et al. (1998) Prevalence proportion ratios: estimation and hypothesis testing. Int $J$ Epidemiol 27, 91-95.

30. Zou G (2004) A modified Poisson regression approach to prospective studies with binary data. Am J Epidemiol 159, 702-706.

31. Guo X, Pan W, Connett JE, et al. (2005) Small-sample performance of the robust score test and its modifications in generalized estimating equations. Stat Med 24, 3479-3495.

32. Institute of Medicine of the National Academies (2010) Dietary Reference Intakes for Calcium and Vitamin D. Washington, DC: The National Academies Press.

33. Rothman KJ, Moore LL, Singer MR, et al. (1995) Teratogenicity of high vitamin A intake. $N$ Engl J Med 333, 1369-1373.

34. Martinez FD, Wright AL, Taussig LM, et al. (1995) Asthma and wheezing in the first six years of life. The Group Health Medical Associates. N Engl J Med 332, 133-138.

35. Martindale S, McNeill G, Devereux G, et al. (2005) Antioxidant intake in pregnancy in relation to wheeze and eczema in the first two years of life. Am J Respir Crit Care Med 171, 121-128.

36. West CE, Dunstan J, McCarthy S, et al. (2012) Associations between maternal antioxidant intakes in pregnancy and infant allergic outcomes. Nutrients 4, 1747-1758.

37. Erkkola M, Nwaru BI, Kaila M, et al. (2012) Risk of asthma and allergic outcomes in the offspring in relation to maternal food consumption during pregnancy: a Finnish birth cohort study. Pediatr Allergy Immunol 23, 186-194.

38. Ellwood P, Asher MI, Bjorksten B, et al. (2001) Diet and asthma, allergic rhinoconjunctivitis and atopic eczema symptom prevalence: an ecological analysis of the International
Study of Asthma and Allergies in Childhood (ISAAC) data ISAAC Phase One Study Group. Eur Respir J 17, 436-443.

39. Farchi S, Forastiere F, Agabiti N, et al. (2003) Dietary factors associated with wheezing and allergic rhinitis in children. Eur Respir J 22, 772-780.

40. Montano Velazquez BB, Jauregui-Renaud K, Banuelos Arias Adel C, et al. (2006) Vitamin E effects on nasal symptoms and serum specific IgE levels in patients with perennial allergic rhinitis. Ann Allergy Asthma Immunol 96, 45-50.

41. Shahar E, Hassoun G \& Pollack S (2004) Effect of vitamin E supplementation on the regular treatment of seasonal allergic rhinitis. Ann Allergy Asthma Immunol 92, 654-658.

42. Baehner RL, Boxer LA, Allen JM, et al. (1977) Autooxidation as a basis for altered function by polymorphonuclear leukocytes. Blood 50, 327-335.

43. Chirico G, Marconi M, Colombo A, et al. (1983) Deficiency of neutrophil phagocytosis in premature infants: effect of vitamin E supplementation. Acta Paediatr Scand 72, 521-524.

44. Wieringa FT, Dijkhuizen MA, West CE, et al. (2004) Reduced production of immunoregulatory cytokines in vitamin A- and zinc-deficient Indonesian infants. Eur J Clin Nutr $\mathbf{5 8}$ 1498-1504

45. Cantorna MT, Nashold FE \& Hayes CE (1995) Vitamin A deficiency results in a priming environment conducive for Th1 cell development. Eur J Immunol 25, 1673-1679.

46. Schuster GU, Kenyon NJ \& Stephensen CB (2008) Vitamin A deficiency decreases and high dietary vitamin A increases disease severity in the mouse model of asthma. J Immunol 180, 1834-1842.

47. Jason J, Archibald LK, Nwanyanwu OC, et al. (2002) Vitamin A levels and immunity in humans. Clin Diagn Lab Immunol 9, 616-621.

48. Huang SL \& Pan WH (2001) Dietary fats and asthma in teenagers: analyses of the first Nutrition and Health Survey in Taiwan (NAHSIT). Clin Exp Allergy 31, 1875-1880

49. de la Rubia J, Grau E, Montserrat I, et al. (1989) Anaphylactic shock and vitamin $\mathrm{K}_{1}$. Ann Intern Med 110, 943.

50. Havel M, Muller M, Graninger W, et al. (1987) Tolerability of a new vitamin $\mathrm{K}_{1}$ preparation for parenteral administration to adults: one case of anaphylactoid reaction. Clin Ther $\mathbf{9}$, 373-379

51. Martin JC (1991) Anaphylactoid reactions and vitamin K. Med J Aust 155, 851.

52. Martinez-Abad M, Delgado F, Palop V, et al. (1991) Vitamin $\mathrm{K}_{1}$ and anaphylactic shock. DICP 25, 871-872.

53. O'Reilly RA \& Kearns P (1995) Intravenous vitamin $\mathrm{K}_{1}$ injections: dangerous prophylaxis. Arch Intern Med 155 $2127-2128$

54. Rich EC \& Drage CW (1982) Severe complications of intravenous phytonadione therapy. Two cases, with one fatality. Postgrad Med 72, 303-306.

55. Chung JY, Ramos-Caro FA, Beers B, et al. (1999) Hypersensitivity reactions to parenteral vitamin K. Cutis 63, 33-34

56. Finkelstein H, Champion MC \& Adam JE (1987) Cutaneous hypersensitivity to vitamin $\mathrm{K}_{1}$ injection. J Am Acad Dermatol 16, 540-545.

57. Lemlich G, Green M, Phelps R, et al. (1993) Cutaneous reactions to vitamin $\mathrm{K}_{1}$ injections. $J$ Am Acad Dermatol 28, 345-347.

58. Arvaniti F, Priftis KN, Papadimitriou A, et al. (2011) Adherence to the Mediterranean type of diet is associated with lower prevalence of asthma symptoms, among 10-12 years old children: the PANACEA study. Pediatr Allergy Immunol 22, 283-289. 
59. Tabak C, Wijga AH, de Meer G, et al. (2006) Diet and asthma in Dutch school children (ISAAC-2). Thorax 61, 1048-1053.

60. Wijga AH, Smit HA, Kerkhof M, et al. (2003) Association of consumption of products containing milk fat with reduced asthma risk in pre-school children: the PIAMA birth cohort study. Thorax 58, 567-572.

61. Willers SM, Wijga AH, Brunekreef B, et al. (2011) Childhood diet and asthma and atopy at 8 years of age: the PIAMA birth cohort study. Eur Respir J 37, 1060-1067.

62. Bel EH (2004) Clinical phenotypes of asthma. Curr Opin Pulm Med 10, 44-50.

63. Henderson J, Granell R \& Sterne J (2009) The search for new asthma phenotypes. Arch Dis Child 94, 333-336.
64. Stein RT \& Martinez FD (2004) Asthma phenotypes in childhood: lessons from an epidemiological approach. Paediatr Respir Rev 5, 155-161.

65. Braun-Fahrlander C, Wuthrich B, Gassner M, et al. (1997) Validation of a rhinitis symptom questionnaire (ISAAC core questions) in a population of Swiss school children visiting the school health services. SCARPOL-team. Swiss Study on Childhood Allergy and Respiratory Symptom with respect to Air Pollution and Climate. International Study of Asthma and Allergies in Childhood. Pediatr Allergy Immunol 8, 75-82.

66. Wuthrich B, Schindler C, Leuenberger P, et al. (1995) Prevalence of atopy and pollinosis in the adult population of Switzerland (SAPALDIA study). Swiss Study on Air Pollution and Lung Diseases in Adults. Int Arch Allergy Immunol 106, 149-156. 